\title{
Menopause and cardiovascular risk
}

\author{
R. Rossi, T. Grimaldi, G. Origliani, G. Fantini, F. Coppi, M.G. Modena \\ Cattedra di Cardiologia, Università di Modena e Reggio Emilia
}

\begin{abstract}
Menopause is not a disease, but a physiologic phase of a woman's life, due to the changes of their hormonal status. Fastidious symptoms may be associated with changes in the metabolism together with new cardiovascular risk factors, particularly aggressive for the female cardiovascular system, unprepared because of the protection due to the fertile period. Changes of the lipid profile, obesity, hypertension, glucose intolerance and diabetes mellitus may intervene as severe risk factors. Cardiovascular disease represents therefore the most frequent cause of mortality and morbidity also in the female gender more than cancer either in the United States as in Europe. The risks related to postmenopause are mainly due to the abrupt interruption of estrogen, which has indirect protective effects on lipid, glycidic metabolism and direct effects on vessel function. They have, in fact, vasodilator action due to nitric oxide release, calcium-antagonist like action and an antiproliferative effect on smooth muscle cells. Postmenopause is also frequently associated with hypertension, the most frequent related factor to coronary artery disease. Hypertension is due to increased body mass index, with insulin-resistance, sodium retention, increased blood viscosity and estrogen deficiency with increased smooth muscle cell proliferation which deter-
\end{abstract}

(c) 2002 S. Karger AG. Basel

$1424-8832 / 02 / 0326-0325 \$ 18.50 / 0$

Fax +4161306 1234 mines an increase in systemic vascular resistance. Age and estrogen deficiency are together the most important cause of cardiovascular risk in post-menopause. The discovery of alpha and recently beta estrogen receptors on coronary female vessels unaffected by atherosclerosis either during pre and post-menopause phase are possible key of interpretation of pathophysiology of coronary artery disease in women, with important therapeutic consequence.

Copyright (C) 2002 S. Karger AG. Basel

Menopause is not "per se" a disease, but can be a risk factor. Its onset may be accompanied by psychological and physical symptoms only, able nevertheless to influence quality of life of women. Quite all women moreover are affected by metabolic modifications which, sometimes, become a real cardiovascular risk profile, practically unknown trough all the fertile life. This consists of a totally new risk profile, which intervenes rapidly without the so-called preconditioning phenomenon; this implies an aggressive influence on a unprepared organism. This metabolic high risk syndrome consists of hypertension, dislipidemia, obesity and insuline-resistance o real diabetes.

We would like to discuss, on this report, our experience on menopause and risk factors, focusing on our research mainly dedicated diabetes, hypertension, hormone replacement therapy influence, achieved by the Center active at our Institute

Professor Maria Grazia Modena

Cardiologia - Azienda Ospedaliera - Policlinico di Modena

tel. +39-059-4224241, fax 039-059-4224323

E-Mail: modena.mariagrazia@unimo.it 
from 1996, dedicated to post-menopausal women. Diabetes is the most important risk factor in women, much higher than in men. In the Nurses' Health Study diabetes was associated to an increased cardiovascular risk from 3 to 7 fold. Diabetes moreover may cause a microvascular disease with a consequent silent ischemic heart disease and diastolic failure, typical of elderly women. Diabetic women enter severe complications also in myocardial revascularization procedures when diabetes (a well-known cause of endothelial dysfunction, micro and macro-angiopathy, vessel calcifications) is added to the anatomical female condition of small, fragile coronary and peripheral arteries. We are performing at our Institute a study on diabetic women (aged 40-65 years) with a 10 year followup. This study started 5 years ago and the flow-chart of our approach is represented in Figure 1. All high risk diabetic women have been moreover enrolled in a new study protocol with Cardiac-CT either for calcium score evaluation as well as for virtual angiography and angioscopy.

Another leading problem in Postmenopause is obesity and adipose tissue redistribution. There is certainly a direct relationship between body weight and all cause morbidity and mortality. There are data from the US National Center of Health Statistics (1991) showing that $50 \%$ of black and about $33 \%$ of Caucasian women in the US have a body weight overcoming the $20 \%$ of the ideal weight. Obesity is affecting especially black, low social level, hispanic women but is becoming a real problem also in our country. Data from the National Health Institute show a high prevalence of overweight and obesity in the south of Italy. Obesity is "per se" a risk factor because of the influence on glicidic metabolism, insulineresistance, blood pressure and lipid profile. For this reason although the failure of hormonal replacement therapy (HRT) in either primary as well, and more, secondary prevention, we have recently started a trial on HRT in women with signs and

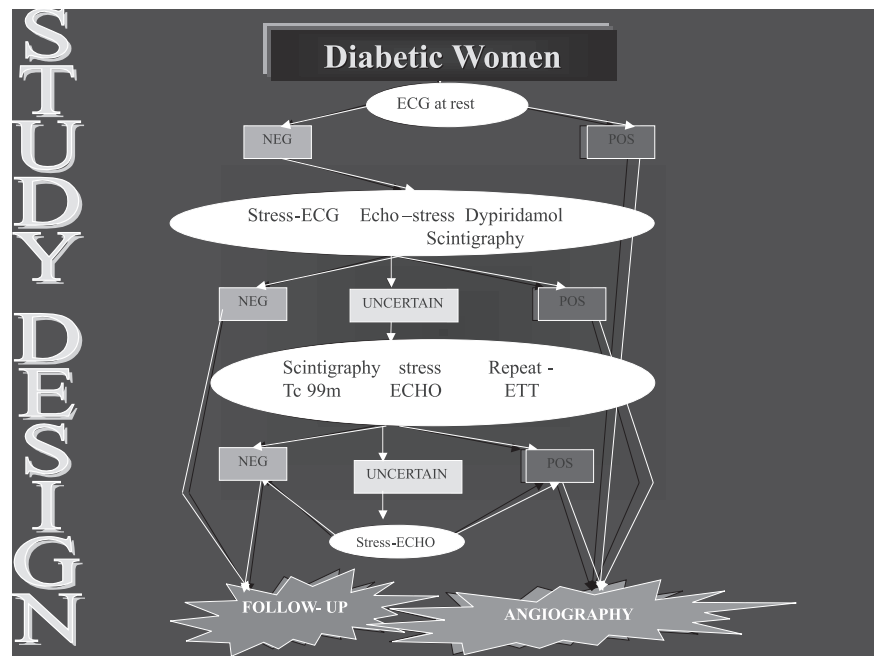

Fig. 1. Flow-chart adopted in the evaluation of diabetic women at our Institute. symptoms of metabolic syndrome, coordinated by our Center.

A report of the European Union has stated that smoking, alcoholism, stress, overweight and the absence of physical activities are new important risk factors. In Italy smoking is an increasing risk factor for incidence $(42.3 \%)$ but the highest prevalence is reported from Portugal. In our Country stress is also important $(24.6 \%)$ and we are overcome only by Greece, the Netherlands and Sweden. Finally hypertension is probably the most important risk factor in women either for incidence, prevalence as well as for morbidity and mortality.

At this point we would like to discuss hypertension in women since it represents our main field of clinical research. The JNC [1] does not consider hypertension as a disease, but as a risk factor, which increases the risk in some organs, defined target organ of hypertension, to undergo reversible or not reversible modification. The target organ damage is therefore the result of the interaction of high blood pressure with other risk factors and this is particularly important in Postmenopause women. Among so-called target organ damage, left ventricular hypertrophy represents the most interesting one, also because the only sometimes really reversible. All randomized and observational studies agree about the issue that smaller is left ventricular mass (LVM) less are the risk of stroke, heart failure and coronary events. The reduction in LVM is also associated to a better prognosis. From several metanalisis we know that LVM may be easily reduced with many drugs, or quite all active antihypertensive treatments. We have tested, sometimes ago, if also HRT could be helpful in increasing the effect of antihypertensive therapy. We investigated the effects of transdermal 17b-estradiol, combined with standard antihypertensive therapy, on the modification of the cardiovascular risk profile in hypertensive postmenopausal women [2]. In a randomized, double-blind, placebo-controlled study, we enrolled 200 postmenopausal women with mild to moderate hypertension. Patients received $17 \mathrm{~b}$-estradiol $(50$ $\mathrm{mg} /$ day, transdermal) and norethisterone acetate $(2.5 \mathrm{mg} /$ day, orally) or placebo. At baseline serum total cholesterol, LDL cholesterol, HDL cholesterol, triglycerides, glucose and fibrinogen plasma levels were measured and all subjects underwent complete M-mode and 2-D echocardiograms, which were repeated after 6,12 and 18 months of HRT. Compared with placebo, all values decreased significantly except for HDL cholesterol. In both groups no modifications were observed in echocardiographic parameters, except for left ventricular mass index, which showed a significant decrease in both groups. The reduction was greater in the treated group; the percentage of patients with left ventricular hypertrophy was $46 \%$ before randomization and $17.2 \%$ after 18 months of treatment $(\mathrm{p}<0.0001)$ whereas in group II the percentage was $48 \%$ at baseline and $31.5 \%$ after 18 months $(\mathrm{p}<0.05)$. Our conclusion were that transdermal $17 \mathrm{~b}$-estradiol, associated to antihypertensive therapy, may contribute to the reduction of cardiovascular risk profile in hypertensive postmenopausal 
women. In a subsequent study [3] we decided to speculate more on the aspect of possible regression of LVM and systolic performance in hypertensive postmenopausal women using transdermal 17b-estradiol, combined with standard antihypertensive therapy. In a randomized, double-blind, placebo-controlled study, we enrolled 169 postmenopausal women with mild or moderate hypertension. Eighty-six patients (group 1) received transdermal $17 \mathrm{~b}$-estradiol $(50 \mathrm{mg} / \mathrm{die})$ and norethisterone acetate $(2.5 \mathrm{mg} / \mathrm{d}$, orally), and 83 patients (group 2) received placebo. At baseline all women underwent M-mode and 2-D echocardiogram, which was repeated after 6, 12 and 18 months of follow-up. After 18 months of treatment we observed a significant decrease in left ventricular diastolic septal and posterior wall thickness and mass in both groups. Furthermore, after 18 months, left ventricular mass was significantly less in the estrogen-treated group. No significant modifications were observed in left ventricular systolic and diastolic dimensions or in systolic performance, as expressed by left ventricular fractional shortening. Those results appeared interesting, but insufficient in solving our current problems in hypertensive women for two reasons: only a low percentage assume HRT for their own skepticism and also general practitioners are quite adverse and disagree with the opinion of gynecologists, who too often recommend HRT. For example in our region only $10-15 \%$ of post-menopausal women assume estrogens, half of them for less than 1 year and only a small percentage for a useful time (4-5 years) in terms of prevention and therapy. The association therefore of antihypertensive treatment to HRT, although efficacious, does not represent, statistically, an useful impact on the target organ damage. Our approach has consequently been moved toward a more large and sophisticate tool, in other words on the treatment of the socalled endothelial dysfunction, since it is well known its involvement in the pathogenesis of the atherosclerotic process. All risk factors moreover are responsible of endothelial dysfunction which may represents the mean through which hypertension induces hypertension associated disease and determines target organ damage. Several recent studies have shown that endothelial function may be evaluated with a noninvasive ultrasonic technique, with optimal correlation with the standard invasive method. We are adopting this technique for studying vascular reactivity in post menopause women with different risk factors. Particularly our group tested [4] if there is a blood pressure lowering agent superior to others in improving endothelial function in a population of hypertensive postmenopausal women treated with a single-drug therapy. We enrolled 106 postmenopausal women with mild to moderate hypertension (diastolic blood pressure from 95 to $110 \mathrm{mmHg}$ ). They received: diuretic (Group I, $\mathrm{n}=19$ ), b-blocker (Group II, $\mathrm{n}=21$ ), calcium antagonist (Group III, $\mathrm{n}=31$ ) or ACEinhibitor (Group IV, $\mathrm{n}=35$ ). Patients achieving a diastolic blood pressure of $<90 \mathrm{mmHg}$ during drug titration, entered a 6 month maintenance period. We assessed, at baseline and after 6 months of treatment, flow-mediated endothelium dependent vasodilation (EdVD) by measuring the diameter of the brachial artery before and during reactive hyperemia (induced after deflation of a blood pressure cuff inflated to suprasystolic pressure for 5 minutes). There were no baseline intergroup differences regarding age, cardiovascular risk factors and use of hormonal replacement therapy. After 6 months of therapy, EdVD was significantly greater in patients treated with ACE-inhibitors (Group IV) compared to other groups. It seems that ACE-inhibitors, in postmenopausal hypertensive women, appeared to better contribute to improve endothelial function. More recently we tried to test the prognostic value of endothelial dysfunction reversibility. For this reason we studied 175 consecutive postmenopausal women (age $61 \pm 5$ years) with newly diagnosed mild to moderate hypertension, that underwent repeat echocardiographic study of the brachial artery 6 months after the initial examination, while optimal control of blood pressure (blood pressure $<140 / 90 \mathrm{mmHg}$ ) was achieved using antihypertensive therapy and were then followed up for a mean period of 5.1 years (range 4.1-5.4 years). Flow-mediated EdVD was evaluated by measuring the diameter of the brachial artery before and during reactive hyperemia (induced after deflation of a blood pressure cuff inflated to suprasystolic pressure for 5 minutes). After 6 months of antihypertensive treatment EdVD had not changed ( $£ 10 \%$ respect to baseline) in 75 of 175 women (42.8\%) (group I), whereas it was significantly improved ( $>10 \%$ respect to baseline) in the remaining 125 women (57.2\%) (group II). Baseline EdVD was similar in the two groups and there were no intergroup differences regarding age, prevalence of cardiovascular risk factors and use of hormone replacement therapy. By Cox model analysis, a significant improvement of EdVD (> 10\% respect baseline) emerged as the single best predictor of cardiovascular events (chi-square 15.70). In this study we demonstrated that a significant improvement of EdVD may be obtained after 6 months of antihypertensive therapy and clearly identifies patients with more favorable outcome [5].

Finally we have investigated in 83 women the interaction between estrogens and C-Reactive protein (CRP), since we are firmly convinced that hormone have an important biological role probably to be tested with different dosages and route of administration. We have in fact in this group of women recently demonstrated that transdermal $17 \mathrm{~b}$ estradiol reduced, whereas oral estrogen increased CRP [6].

After these considerations, it may be underlined that cardiovascular disease is the main cause of morbidity and mortality in the West Countries, much more important than cancer of all causes. In the US the annual mortality for cardiovascular disease is $49 \%$, in Europe $43 \%$. Nevertheless the highest incidence of coronary artery disease in the male population has generated the concept that female gender was immune from atherosclerosis. Nowadays new researches have allowed to the consciousness that women's mortality for cardiovascular dis- 
ease is the same of men. The only real difference consists in the fact that women have risk profile, clinical manifestation and natural history of the disease totally peculiar. This peculiarity is probably due to estrogens and to other undefined biological influence on the cardiovascular system in the female gender, that need to be investigated. Recent trial on HRT has shocked the scientific arena, but we firmly believe that women should be further studied from the biological and hormonal point of view, because something, still unknown, distinguish gender and characterizes women's peculiarity.

\section{References}

1. The Fifth Report of the Joint National Committee on detection, evaluation and treatment of high blood pressure (JNC V). Arch Intern Med. 1993; 153: 154-291

2. Modena MG, Castelli A, Rossi R, et al. Double-blind randomized placebo-controlled study of transdermal estrogen replacement therapy on hypertensive postmenopausal women. Am J Hypertens 1999; 12: 1000-1008

3. Modena MG, Aveta P, Rossi R, et al. Effects of transdermal 17b-estradiol on left ventricular anatomy and performance in hypertensive women. Hypertension. 1999; 34: 1041-1046

4. Rossi R, Aveta P, Modena MG, et al. Effects of single-drug antihypertensive therapy on endothelium-dependent vasodilation in hypertensive postmenopausal women. J Am Coll. Cardiol 2000; 35 (n. 2, Suppl. A): 287 A

5. Maria G Modena, Francesca Coppi, Rosario Rossi, et al. Prognostic Role of Reversible
Endothelial Dysfunction in Hypertensive Postmenopausal Women. JACC Vol. 40, No 3, 2002. August 7, 2002: 505-10

6. Maria G. Modena, Francesca Bursi, Rosario Rossi, et al. Effects of hormone replacement therapy on C-Reactive protein levels: differences between oral and transdermal administration of estrogens. Am J Med. In press 